\title{
Thermopower of a single electron transistor in the regime of strong inelastic cotunneling
}

\author{
K. A. Matveev ${ }^{1}$ and A. V. Andreev ${ }^{2,3}$ \\ ${ }^{1}$ Department of Physics, Duke University, Box 90305, Durham, NC 27708 \\ ${ }^{2}$ Department of Physics, University of Colorado, CB 390, Boulder, CO 80309 \\ ${ }^{3}$ Bell Labs, Lucent Technologies, 600 Mountain Ave., Murray Hill, NJ 07974
}

(January 11, 2002)

\begin{abstract}
We study Coulomb blockade oscillations of thermoelectric coefficients of a single electron transistor based on a quantum dot strongly coupled to one of the leads by a quantum point contact. At temperatures below the charging energy $E_{C}$ the transport of electrons is dominated by strong inelastic cotunneling. In this regime we find analytic expressions for the thermopower as a function of temperature $T$ and the reflection amplitude $r$ in the contact. In the case when the electron spins are polarized by a strong external magnetic field, the thermopower shows sinusoidal oscillations as a function of the gate voltage with the amplitude of the order of $e^{-1}|r| \frac{T}{E_{C}}$. We obtain qualitatively different results in the absence of the magnetic field. At temperatures between $E_{C}$ and $E_{C}|r|^{2}$ the thermopower oscillations are sinusoidal with the amplitude of order $e^{-1}|r|^{2} \ln \frac{E_{C}}{T}$. On the other hand, at $T \ll E_{C}|r|^{2}$ we find non-sinusoidal oscillations of the thermopower with the amplitude $\sim e^{-1}|r| \sqrt{T / E_{C}} \ln \left(E_{C} / T\right)$.
\end{abstract}

PACS numbers: 73.23.Hk, 73.50.Lw, 72.15.Jf

\section{INTRODUCTION}

It is well known that electric current in solids can be caused not only by an applied electric field, but also by a temperature gradient. This gives rise to a number of interesting thermoelectric phenomena. to note that the thermoelectric phenomena in metals require asymmetry between electrons and holes. Indeed, in a perfectly electron-hole symmetric system the temperature gradient will cause equal in magnitude currents of electrons and holes which result in zero net electric current.

Many recent studies of thermpolectric effects were focused on mesoscopic systems. 111 Thermoelectric properties of these systems are particularly interesting because the electron-hole asymmetry in mesoscopic devices is usually strong and can be controlled experimentally by tuning external parameters, such as gate voltage or magnetic field.

Of the various thermoelectric phenomena the Peltier effect is probably the most important for technological applications: when an electric current $I$ is passed through a system in the absence of the temperature gradient, it is accompanied by the heat current

$$
I_{Q}=\Pi I .
$$

Here $\Pi$ is the Peltier coefficient. The use of the Peltier effect has been proposed for refrigeration in conditions when various technological constraints, such as the size of the device, outweigh the power efficiency considerations. The strong enhancement of the particle-hole asymmetry in mesoscopic devices and their small size make them very promising candidates for microrefrigerators. 0
In the last few years many experimental and theoretical studies 11 focused on the thermoelectric properties of single electron transistors (SET). Thermoelectric effects in these systems can be controlled by the gate voltage $V_{g}$, Fig. 1, and exhibit characteristic Coulomb blockade oscillations. Most of the studies of thermoelectric effects in the Coulomb blockade regime concentrated on the thermopower

$$
S=-\left.\frac{V}{\Delta T}\right|_{I=0} .
$$

Here $V$ is the voltage induced across the device in the absence of net electric current when the temperatures of the two leads differ by $\Delta T$, Fig. 1 1 (a). The Peltier coefficient $\Pi$ is related to the thermopower $S$ by an Onsager relation $\Pi=S T$.

The theory of the Coulomb blockade oscillations in the thermopower of single electron transistors in the weak tunneling regime was developed in Ref. 7. This theory takes into account only the lowest order tunneling processes, i.e. the sequential tunneling, and neglects the cotunneling processes. Its results mere in agreement with the experiments of Ref. 8. Latere it became possible to experimentally access the regime of lower temperatures and stronger coupling to the leads, where the cotunneling processes become dominant. The theoretical description of this regime was recently given in Ref. 11 .

In experiments with GaAs heterostructures the quantum dot is connected to the leads by quantum point contacts. Each contact is usually in the regime when only one transverse mode can propagate through it, and the transmission coefficient for this mode can be controlled experimentally. Recently 10 the Coulomb blockade oscillations in the thermopower of a SET with the quantum 
dot strongly coupled to one of the leads were studied for various values of the reflection coefficient $|r|^{2}$ in this contact. The setup of these experiments is schematically shown in Fig. 1(b). In the regime of strong coupling, $|r|^{2} \ll 1$, nearly sinusoidal oscillations of the thermopower as a function of the gate voltage $V_{g}$ were observed.
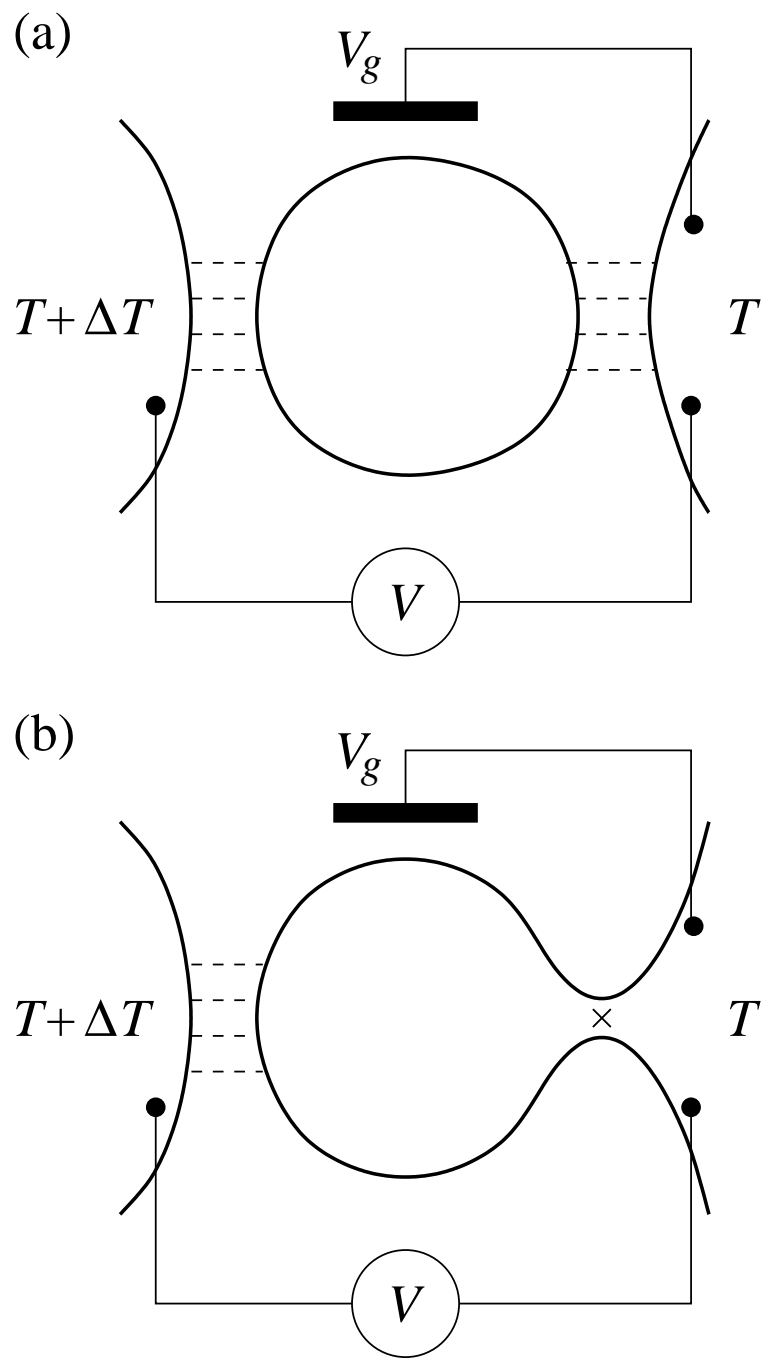

FIG. 1. (a) Setup of the thermopower measurement in a single electron transistor. A quantum dot is capacitively coupled to the gate and connected to the two leads by tunneling junctions. The left and right leads are maintained at temperatures $T+\Delta T$ and $T$, respectively, and the voltage $V$ across the device is measured. The thermopower (2) is measured as a function of the gate voltage $V_{g}$. (b) A SET with a quantum dot strongly coupled to the right lead by a single-channel quantum point contact. The cross $\times$ in the constriction represents the backscattering in the contact resulting in a finite reflection coefficient $|r|^{2}$.

The theories of thermopower for the weak tunneling regime developed in Refs. 6,11 rely upon the perturbation theory in the strength of coupling between the quantum dot and the leads. This perturbative approach fails when the coupling to the leads is strong. The previous theoretical work on Coulomp blockade systems with strongly coupled quantum dots 1215 was devoted to the studies of their thermodynamic properties and conductance. These properties are not sensitive to the electron-hole asymmetry, and the calculation of the thermoelectric properties requires a non-trivial generalization of the approach of Refs. 14,15.

In this paper we develop a theory of the thermopower in a SET with a quantum dot strongly coupled to one of the leads, Fig. 1(b). We consider a relatively large quantum dot in which the quantum level spacing is small, and the electron transport is dominated by inelastic cotunneling. In Sec. II we present a qualitative discussion of thermoelectric transport in a SET. The thermopower of a SET in the regime of strong inelastic cotunneling is developed in Sec. III for the simpler case of spin-polarized electrons as well as for the more interesting spin-degenerate case. We discuss the results and compare them with experiments in Sec. IV.

\section{QUALITATIVE DISCUSSION OF THE THERMOPOWER AT WEAK INELASTIC COTUNNELING}

The physical meaning of the thermopower can be deduced from the Onsager relation $S=\Pi / T$. One can easily see from Eq. (11) that the Peltier coefficient is determined by the average energy $\langle\epsilon\rangle$ of the electrons carrying current through the system, measured from the chemical potential: $\Pi=-\langle\epsilon\rangle / e$. Thus the thermopower measures the average energy of the tunneling electrons in units of the temperature:

$$
S=-\frac{\langle\epsilon\rangle}{e T} .
$$

Here $e$ is the absolute value of the electron charge. The average energy of the charge carriers $\langle\epsilon\rangle$ is determined by a particular mechanism of transport through the system.

A conventional SET schematically shown in Fig. 1 (a) consists of a quantum dot weakly coupled to two leads. The transport of electrons from the left lead to the right one is achieved by either sequential tunneling or cotunneling (elastic or inelastic).

The sequential tunneling refers to the lowest-order tunneling processes in which one electron tunnels into or out of the dot. As a result of each tunneling event the charge of the dot changes by $\pm e$. When an electron tunnels into or out of the dot, the electrostatic energy of the system increases by $u_{+}$or $u_{-}$, respectively. The values of $u_{+}$and $u_{-}$are of the order of the charging energy $E_{C}=e^{2} / 2 C$, where $C$ is the capacitance of the dot; their values can be tuned by adjusting the gate voltage $V_{g}$. The electron that tunnels into the dot has to have the energy $\epsilon \approx u_{+}$in order to charge the dot, so the tunneling is exponentially 
suppressed at low temperatures as $e^{-u_{+} / T}$. Similarly the rate of tunneling out of the dot is suppressed as $e^{-u_{-} / T}$. The thermopower of a SET in the regime of sequential tunneling was studied in Ref. 7.

The cotunneling mechanism accounts for the coherent second-order tunneling processes in which at the first step an electron tunnels from, say, the left lead into the dot, and at the second step this or another electron (for elastic and inelastic cotunneling, respectively) tunnels from the dot to the right lead. Since as a result of cotunneling processes the charge of the dot remains unchanged, the electrons participating in the transport through the dot do not need to have large energies $\sim u_{ \pm}$, and the transport is not suppressed exponentially at low temperatures. It is important to note that the elastic cotunneling involves elastic propagation of electrons between the tunneling contacts in the dot. The resulting contribution to the transport is inversely proportional to the volume of the dot. Thus in relatively large dots the finite temperature transport is dominated by inelastic cotunneling processes.

In order to find the cotunneling thermopower of a SET, one needs to evaluate the average energy $\langle\epsilon\rangle$ of the electron transferred through the device. Inelastic cotunneling involves electrons within the energy strip of width $\sim T$ near the Fermi level. The probability $w(\epsilon)$ of the second-order tunneling process is inversely proportional to the square of the energy of the virtual state,

$$
w(\epsilon) \propto\left(\frac{1}{u_{+}+\epsilon^{\prime}-\epsilon}+\frac{1}{u_{-}+\epsilon-\epsilon^{\prime}}\right)^{2},
$$

where we assumed that the electron with energy $\epsilon$ tunnels into a state of energy $\epsilon^{\prime}$ in the dot. The energies $\epsilon$ and $\epsilon^{\prime}$ are of the order of $T$ and small compared to the charging energies $u_{ \pm}$. One can therefore expand Eq. (4) in small $\left(\epsilon-\epsilon^{\prime}\right) / u_{ \pm}$,

$$
w(\epsilon) \propto\left(\frac{1}{u_{+}}+\frac{1}{u_{-}}\right)^{2}\left[1+2\left(\frac{1}{u_{+}}-\frac{1}{u_{-}}\right)\left(\epsilon-\epsilon^{\prime}\right)\right] .
$$

The $\epsilon$-dependent correction in Eq. (5) shows that the tunneling probability increases or decreases with the energy $\epsilon$ of the tunneling particle depending on the sign of $\left(u_{+}^{-1}-u_{-}^{-1}\right)$. Since the typical energy $\epsilon \sim T$, the relative magnitude of the term breaking the electron-hole symmetry in (5) is $\sim T\left(u_{+}^{-1}-u_{-}^{-1}\right)$ and the average energy of the tunneling particles is $\langle\epsilon\rangle \sim T^{2}\left(u_{+}^{-1}-u_{-}^{-1}\right)$. Then using Eq. (3) we estimate the thermopower of a SET as

$$
S=\lambda \frac{T}{e}\left(\frac{1}{u_{-}}-\frac{1}{u_{+}}\right) .
$$

Here $\lambda$ is a numerical coefficient of order unity; its value $\lambda=4 \pi^{2} / 5$ has been found in Ref. 11 .

An interesting feature of the result (6) is that the thermopower does not depend on the strength of coupling of the dot to the leads. This feature is expected to persist as long as the transmission coefficients of the barriers are small. However, as the barrier approaches the regime of perfect transmission, the Coulomb blockade oscillations of physical quantities are expected to disappear.13.14 One should therefore expect that in the regime of strong coupling the thermopower will depend on the reflection coefficient $|r|^{2}$ of the contact.

It is also worth mentioning that the thermopower $S \sim e^{-1} T / E_{C}$ in a SET is much greater than the typical value $S \sim e^{-1} T / E_{F}$ of the thermopower in metals; here $E_{F}$ is the Fermi energy. This is a consequence of the fact that the charging effects in the dot enhance the electron-hole asymmetry. We will show in Sec. III that such behavior of the thermopower at $T \rightarrow 0$ persists in the strong coupling regime.

\section{THERMOPOWER IN THE REGIME OF STRONG INELASTIC COTUNNELING}

In this section we calculate the thermopower $S$ of a SET in which one of the contacts is in the strong tunneling regime, Fig. 1(b). We restrict ourselves to the linear response regime when both the temperature difference between the leads $\Delta T$ and the voltage $V$ across the device are small. The thermopower (2) is defined in terms of the voltage $V$ induced across the device by the temperature difference $\Delta T$ at zero current. In practice, however, it is easier to calculate the current response

$$
I=G V+G_{T} \Delta T .
$$

Here $G$ is the conductance of the SET, and $G_{T}$ is the thermoelectric coefficient describing the current response to an applied temperature difference. The thermopower (2) can then be expressed as

$$
S=\frac{G_{T}}{G} .
$$

The conductance $G$ of a SET in the regime of strong inelastic cotunneling was found in Ref. 14. Hence in the following we concentrate on the calculation of the thermoelectric coefficient $G_{T}$.

\section{A. Tunneling approximation}

We assume that the conductance of the tunneling junction connecting the dot to the left lead is much smaller than the conductance quantum, $G_{L} \ll e^{2} / h$. In this case one can describe the SET by the Hamiltonian $H=H_{L}+H^{\prime}$, where $H_{L}$ is the Hamiltonian describing the tunneling of electrons in the left contact,

$$
\begin{aligned}
H_{L}= & \sum_{k} \epsilon_{k} a_{k}^{\dagger} a_{k}+\sum_{p} \epsilon_{p} a_{p}^{\dagger} a_{p} \\
& +\sum_{k p}\left(t_{k p} a_{k}^{\dagger} a_{p}+t_{k p}^{*} a_{p}^{\dagger} a_{k}\right),
\end{aligned}
$$


and $H^{\prime}$ accounts for the transport through the right junction and the electron-electron interactions in the system. Here $a_{k}$ and $a_{p}$ are the electron annihilation operators in the left lead and in the dot, respectively, $\epsilon_{k}$ and $\epsilon_{p}$ are the energies of the corresponding states, the matrix elements $t_{k p}$ describe the weak tunneling of the electrons through the barrier.

We will account for the tunneling through the left barrier in the lowest (second) order of the perturbation theory in $t_{k p}$. In this approximation all of the temperature drop occurs at the tunneling barrier. We take the temperature of the left lead to be $T+\Delta T$ and that of the dot to be $T$. Let us denote the tunneling density of states in the left lead by $\nu_{l}(\epsilon)$ and that in the dot by $\nu(\epsilon)$. To the lowest order in the tunneling matrix element the current through the tunneling contact can be obtained with the aid of the Fermi golden rule:

$$
I=-2 \pi e\left\langle\left|t_{k p}\right|^{2}\right\rangle \int_{-\infty}^{\infty} \nu_{l}(\epsilon) \nu(\epsilon)\left[n_{l}(\epsilon)-n(\epsilon)\right] d \epsilon .
$$

Here $n_{l}(\epsilon)$ and $n(\epsilon)$ denote the Fermi distribution functions at the temperature of the left lead $T+\Delta T$ and the $\operatorname{dot} T$, respectively. The square of the tunneling matrix element in Eq. (10) is averaged over the states near the Fermi level.

To determine $G_{T}$ we assume that the chemical potentials in the Fermi functions in Eq. (10) are the same and expand the current to first order in $\Delta T$,

$$
I=-2 \pi e \nu_{l}\left\langle\left|t_{k p}\right|^{2}\right\rangle \frac{\Delta T}{4 T^{2}} \int_{-\infty}^{\infty} \frac{\epsilon \nu(\epsilon)}{\cosh ^{2}\left(\frac{\epsilon}{2 T}\right)} d \epsilon .
$$

Here we have replaced the density of states in the left contact $\nu_{l}(\epsilon)$ by its value $\nu_{l}$ at the Fermi energy using its weak energy dependence. The corrections to this approximation are small in the ratio of the temperature to the Fermi energy. We will see below that the density of states in the dot has the energy dependence at the much smaller energy scale $T$ due to the electron-electron interactions.

In order to express the thermoelectric coefficient $G_{T}$ in terms of physically measurable quantities, we use Eq. (10) to calculate the conductance $G_{L}$ of the left barrier assuming that the electrons in the dot are noninteracting. This conductance can, in principle, be measured experimentally by opening completely the constriction connecting the dot to the right lead. The result is expressed in terms of the density of states in the dot $\nu_{0}$, which is no longer renormalized by the electron-electron interactions:

$$
G_{L}=2 \pi e^{2} \nu_{l} \nu_{0}\left\langle\left|t_{k p}\right|^{2}\right\rangle
$$

Using Eqs. (12) and (11) we can now express $G_{T}=I / \Delta T$ as

$$
G_{T}=-\frac{G_{L}}{4 T^{2} e \nu_{0}} \int_{-\infty}^{\infty} \frac{\epsilon \nu(\epsilon)}{\cosh ^{2}\left(\frac{\epsilon}{2 T}\right)} d \epsilon
$$

Equation (13) reduces our problem to the calculation of the energy-dependent tunneling density of states $\nu(\epsilon)$. We note that unlike the conductance $G$ of the SET, the thermoelectric coefficient $G_{T}$ is determined by the odd (in energy) component of density of states $\nu(\epsilon)$. Therefore the thermopower measurements represent an independent test of the theory of Coulomb blockade in nearly open dots developed in Refs. 13 15.

It is well known that the tunneling density of states can be expressed in terms of the electron Green's function. A specific form of this relation that will be convenient for further calculations is

$$
\nu(\epsilon)=-\frac{1}{\pi} \cosh \frac{\epsilon}{2 T} \int_{-\infty}^{\infty} \mathcal{G}\left(\frac{1}{2 T}+i t\right) \exp (i \epsilon t) d t .
$$

Here $\mathcal{G}(\tau)=-\left\langle T_{\tau} \psi_{L}(\tau) \psi_{L}^{\dagger}(0)\right\rangle$ is the Matsubara Green's function; $\psi_{L}$ is the annihilation operator of an electron in the dot at the position of the left contact. For the derivation of Eq. (14) see Appendix A. Substituting Eq. (14) into Eq. (13), we express the thermoelectric coefficient $G_{T}$ in terms of the Green's function:

$$
G_{T}=\frac{i \pi G_{L}}{2 e \nu_{0}} \int_{-\infty}^{\infty} \frac{\sinh (\pi T t)}{\cosh ^{2}(\pi T t)} \mathcal{G}\left(\frac{1}{2 T}+i t\right) d t .
$$

This expression is insensitive to the specific form of the interactions in the quantum dot and its coupling to the right lead. In the following sections we calculate the Green's function $\mathcal{G}(\tau)$ in the strong inelastic cotunneling approximation and find the corresponding value of $G_{T}$.

\section{B. Inelastic cotunneling approximation}

At finite temperature in a sufficiently large dot one can use the approximation of inelastic cotunneling which neglects the possibility of elastic propagation of electrons between the two contacts in the dot. In this case the system can be modeled by the Hamiltonian

$$
H=H_{L}+H_{R}+H_{C}
$$

in which the Hamiltonians $H_{L}$ and $H_{R}$ describe the independent subsystems of electrons propagating through the left and right contacts; in particular $\left[H_{L}, H_{R}\right]=0$. We have discussed the form of $H_{L}$ in section III A, see Eq. (9). For weak inelastic cotunneling $H_{R}$ would have a form similar to Eq. (9). In the case of strong inelastic cotunneling the Hamiltonian $H_{R}$ has a completely different form which will be discussed in sections III C and IIID. Finally, the term $H_{C}$ describes the Coulomb interactions in the dot. At low energies the interactions are adequately accounted for by the charging energy approximation:

$$
H_{C}=E_{C}\left(\hat{n}_{L}+\hat{n}_{R}-N\right)^{2}
$$


Here $\hat{n}_{L}$ and $\hat{n}_{R}$ are the operators of the number of electrons that entered the dot through the left and right contacts, respectively, and $N$ is a dimensionless parameter which is proportional to the gate voltage $V_{g}$.

By definition of the operator $\hat{n}_{L}$ its commutation relations with the fermion operator $\psi_{L}$ defined below Eq. (14) have the form $\left[\psi_{L}, \hat{n}_{L}\right]=\psi_{L}$. For the convenience of the following calculations we will rewrite the charging energy (17) in the form

$$
H_{C}=E_{C}\left(\hat{n}+\hat{n}_{R}-N\right)^{2},
$$

where $\hat{n}$ is an integer-valued operator that commutes with $\psi_{L}$. In order to preserve the commutation relations between $\psi_{L}$ and $H_{C}$ we replace $\psi_{L} \rightarrow \psi_{L} F$, where $F$ is the operator lowering $\hat{n}$ by unity: $[F, \hat{n}]=F$. It is important to note that the substitution $\psi_{L} \rightarrow \psi_{L} F$ does not affect the form of the Hamiltonian $H_{L}$, and the only modification in the discussion of Sec. IIIA is in the definition of the Green's function in Eq. (14),

$$
\mathcal{G}(\tau)=-\left\langle T_{\tau} \psi_{L}(\tau) F(\tau) F^{\dagger}(0) \psi_{L}^{\dagger}(0)\right\rangle .
$$

The operators $\psi_{L}$ and $\psi_{L}^{\dagger}$ now commute with $H_{R}+H_{C}$, whereas $F$ and $F^{\dagger}$ commute with $H_{L}$. Consequently, the Green's function (19) factorizes,

$$
\mathcal{G}(\tau)=-\left\langle T_{\tau} \psi_{L}(\tau) \psi_{L}^{\dagger}(0)\right\rangle\left\langle T_{\tau} F(\tau) F^{\dagger}(0)\right\rangle .
$$

In the new representation the operators $\psi_{L}$ and $\psi_{L}^{\dagger}$ describe non-interacting fermions, whose Green's function is well known. We can then rewrite $\mathcal{G}(\tau)$ as

$$
\begin{aligned}
\mathcal{G}(\tau) & =-\frac{\nu_{0} \pi T}{\sin (\pi T \tau)} K(\tau), \\
K(\tau) & =\left\langle T_{\tau} F(\tau) F^{\dagger}(0)\right\rangle .
\end{aligned}
$$

Substituting Eq. (20) into Eq. (15) we express the thermoelectric coefficient $G_{T}$ of the dot in terms of the correlator $K(\tau)$,

$G_{T}=-\frac{i \pi^{2}}{2} \frac{G_{L} T}{e} \int_{-\infty}^{\infty} \frac{\sinh (\pi T t)}{\cosh ^{3}(\pi T t)} K\left(\frac{1}{2 T}+i t\right) d t$.

Unlike Eq. (15), formula (22) assumes that the transport through the dot is due to the inelastic cotunneling mechanism, and that the electron-electron interactions in the system are completely described by the charging energy (18). On the other hand, the coupling $H_{R}$ of the dot to the right lead is still arbitrary. In particular, Eq. (22) is valid in the case of a metallic grain coupled to the lead by a wide contact supporting many channels.

In this paper we consider the case of a single-channel contact, which is usually realized in semiconductor devices. Depending on the presence of a magnetic field polarizing the spins of the electrons, one has to consider the cases of either spinless or spin- $\frac{1}{2}$ electrons.

\section{Spinless electrons}

We start with the simpler case of spinless electrons. Following Ref. 13,14 we describe the electron transport through the right quantum point contact by a model of one-dimensional fermions. In the case of strong coupling of the dot to the right lead, the charging energy (18) gives rise to non-trivial Coulomb correlations of the motion of electrons through the constriction. It is more convenient to treat the problems of interacting onedimensional electrons in the bosonized representation. Then the Hamiltonian 13, 44 of the right constriction takes the form $H_{R}=H_{R}^{(0)}+H_{R}^{\prime}$, where

$$
\begin{aligned}
H_{R}^{(0)} & =\frac{v_{F}}{2 \pi} \int_{-\infty}^{\infty}\left\{\pi^{2} \Pi^{2}(x)+\left[\partial_{x} \phi(x)\right]^{2}\right\} d x, \\
H_{R}^{\prime} & =-\frac{D}{\pi}|r| \cos [2 \phi(0)] .
\end{aligned}
$$

Here $\phi$ and $\Pi$ are bosonic fields satisfying the commutation relations $[\phi(x), \Pi(y)]=i \delta(x-y)$, the parameter $v_{F}$ is the Fermi velocity of the electrons, $r$ is the reflection amplitude in the constriction, and $D$ is the bandwidth. The regions $x<0$ and $x>0$ in the integral of Eq. (23a) represent the electrons in the dot and in the right lead, respectively. The deviation of the density of one-dimensional electrons from its ground state value is given by $\partial_{x} \phi(x) / \pi$. Thus the number of electrons that have entered the dot through the right constriction is $n_{R}=\phi(0) / \pi$, and the charging energy (18) takes the form

$$
H_{C}=E_{C}\left[\hat{n}+\frac{1}{\pi} \phi(0)-N\right]^{2} .
$$

The advantage of the bosonization approach is that the Coulomb interaction term (23d) is quadratic in the bosonic operator $\phi$, and, therefore, can be treated exactly. On the other hand, the backscattering of electrons in the constriction in the bosonized representation takes the strongly non-linear form (23b). As a result the backscattering can only be accounted for perturbatively, using the small parameter $|r|<1$.

We will calculate the time-ordered correlator $K(\tau)$ defined by Eq. (21) as an imaginary-time functional integral over the bosonic field $\phi$. The operator $F^{\dagger}(0)$ increases $n$ from 0 to 1 at time $t=0$, whereas $F(\tau)$ changes it back to $n=0$ at time $t=\tau$. Therefore $F(0) F^{\dagger}(0)$ in the functional integral can be omitted provided that the operator $\hat{n}$ in the action is replaced by

$$
n_{\tau}(t)=\theta(t) \theta(\tau-t) .
$$

Here $\theta(t)$ is the unit step function. Upon this procedure the correlator $K(\tau)$ is expressed as follows

$$
K(\tau)=\frac{Z(\tau)}{Z(0)},
$$


where $Z(\tau)$ is the functional integral given by

$$
Z(\tau)=\int \exp \left[-\mathcal{S}_{0}-\mathcal{S}_{C}(\tau)-\mathcal{S}^{\prime}\right] \mathcal{D} \phi(x, t) .
$$

Here $\mathcal{S}_{0}$ denotes the part of the Eucledian action derived from the Hamiltonian (23a) of free electrons moving through the constriction in the absence of both interactions and backscattering:

$$
\mathcal{S}_{0}=\int_{0}^{\beta} d t \int d x \frac{v_{F}}{2 \pi}\left[\frac{\left(\partial_{t} \phi\right)^{2}}{v_{F}^{2}}+\left(\partial_{x} \phi\right)^{2}\right] .
$$

Here $\beta=1 / T$. The term $S_{C}(\tau)$ is the part of the action which is due to the charging energy (23d), where the operator $\hat{n}$ is replaced with (24),

$$
\mathcal{S}_{C}(\tau)=\int_{0}^{\beta} E_{C}\left[n_{\tau}(t)+\frac{1}{\pi} \phi(0, t)-N\right]^{2} d t .
$$

Finally, $S^{\prime}$ is the small contribution to the action due to the backscattering (23b),

$$
S^{\prime}=-\int_{0}^{\beta} \frac{D}{\pi}|r| \cos [2 \phi(0, t)] d t .
$$

The following calculations are performed in the regime of low temperatures, $T \ll E_{C}$. At $r=0$ the functional integral (26) is gaussian, and its explicit evaluation gives

$$
K_{0}(\tau)=\left(\frac{\pi^{2} T}{\gamma E_{C}}\right)^{2} \frac{1}{\sin ^{2}(\pi T \tau)},
$$

see Appendix B. Here $\gamma=e^{\mathbf{C}}$, where $\mathbf{C} \approx 0.577$ is Euler's constant. The substitution of (28) into the expression (22) results in $G_{T}=0$, as the integrand is an odd function of $t$. This is a consequence of the fact that at $r=0$ the system described by the Hamiltonian (23a) and (23d) possesses electron-hole symmetry. Indeed, in the absence of backscattering the dependence of the Hamiltonian on the gate voltage $N$ can be removed by shifting the field $\phi(x) \rightarrow \phi(x)+\pi N$. Then the Hamiltonian $H_{R}^{(0)}+H_{C}$ is obviously invariant with respect to the electron-hole symmetry transformation $\hat{n} \rightarrow-\hat{n}$, $\phi \rightarrow-\phi$, and $\Pi \rightarrow-\Pi$. On the other hand, the shift $\phi(x) \rightarrow \phi(x)+\pi N$ will change the backscattering term (23b). Thus the backscattering breaks the electron-hole symmetry and gives rise to non-zero thermopower of the device.

To account for the small backscattering at $|r| \ll 1$ we expand the action in Eqs. (25) and (26) to first order in $S^{\prime}$ and find

$$
K(\tau)=K_{0}(\tau)\left(1-\left\langle\mathcal{S}^{\prime}\right\rangle_{\tau}+\left\langle\mathcal{S}^{\prime}\right\rangle_{0}\right) .
$$

Here $\left\langle\mathcal{S}^{\prime}\right\rangle_{\tau}$ is defined as

$$
\left\langle\mathcal{S}^{\prime}\right\rangle_{\tau}=\frac{\int \mathcal{S}^{\prime} \exp \left[-\mathcal{S}_{0}-\mathcal{S}_{C}(\tau)\right] \mathcal{D} \phi}{\int \exp \left[-\mathcal{S}_{0}-\mathcal{S}_{C}(\tau)\right] \mathcal{D} \phi} .
$$

Due to the form (27c) of the perturbation $\mathcal{S}^{\prime}$ the evaluation of $K(\tau)$ in Eq. (29) again amounts to taking gaussian functional integrals. The straightforward but lengthy calculations carried out in Appendix B give the result

$$
\begin{aligned}
K(\tau)=K_{0}(\tau)[ & 1-2 \gamma \xi|r| \cos (2 \pi N) \\
& \left.+4 \pi^{2} \xi \gamma|r| \frac{T}{E_{C}} \sin (2 \pi N) \cot (\pi T \tau)\right] .
\end{aligned}
$$

Here $\xi \approx 1.59$ is a constant defined by Eq. (B11). The substitution of this result into Eq. (22) gives the following result for the thermoelectric coefficient in the first order in $|r|$,

$$
G_{T}=-\frac{8 \pi^{7} \xi G_{L}}{15 \gamma e}\left(\frac{T}{E_{C}}\right)^{3}|r| \sin (2 \pi N) .
$$

It is interesting to note that the thermoelectric coefficient $G_{T}$ given by Eq. (32) is an odd function of the gate voltage $N$. This property is more general than the perturbative result (32). Indeed, one can see from the form of the functional integral (26) and the action (27) that the correlator (25) has the following symmetry property: $K(\beta-\tau, N)=K(\tau, 1-N)$. Furthermore, all the physical properties of the system are periodic in $N$ with period 1 . This can be shown by shifting $\phi \rightarrow \phi+\pi N$ which removes $N$ from $S_{C}(\tau)$ and changes the cosine in Eq. (27d) to $\cos [2 \phi(0, t)+2 \pi N]$. The action then becomes invariant with respect to the shift $N \rightarrow N+1$. Consequently the correlator (25) has the property $K(\beta-\tau, N)=K(\tau,-N)$. One can easily see from Eq. (22) that only the part of $K(\tau)$ which is odd with respect to $\tau \rightarrow \beta-\tau$ contributes to the thermoelectric coefficient $G_{T}$. Using the aforementioned properties of $K(\tau, N)$, this odd part can be presented as

$$
\begin{aligned}
K_{\text {odd }}(\tau) & =\frac{1}{2}[K(\tau, N)-K(\beta-\tau, N)] \\
& =\frac{1}{2}[K(\tau, N)-K(\tau,-N)] .
\end{aligned}
$$

Therefore the thermoelectric coefficient $G_{T}$ is an odd function of $N$.

The conductance $G$ can be obtained by substitution of correlator (31) analytically continued to real time into Eq. (52) of Ref. 14. The result has the form

$$
G=G_{L} \frac{2 \pi^{4} T^{2}}{3 \gamma^{2} E_{C}^{2}}[1-2 \gamma \xi|r| \cos (2 \pi N)] .
$$

This expression is in agreement with the formula (A27) of Ref. 14, where the numerical prefactor in the brackets was not determined, and with the expression (34) of Ref. 16, where the constant $\xi$ was found.

Substituting Eq. (32) into Eq. (8) and using the leading term in Eq. (34) for the conductance, we obtain the following expression for the thermopower in the spinless case 


$$
S=-\frac{4 \pi^{3} \xi \gamma T}{5 e E_{C}}|r| \sin (2 \pi N) .
$$

It is instructive to compare this result with the thermopower (6) in the regime of weak inelastic cotunneling. Both expressions vanish linearly at $T \rightarrow 0$, but unlike Eq. (6), our result (35) depends on the transmission coefficient of the barrier. As expected, at perfect transmission $r \rightarrow 0$ the Coulomb blockade oscillations of the thermopower disappear.

\section{Electrons with spin}

Although the spins of electrons can be polarized in an experiment by applying a strong magnetic field, the most common situation is when no field is applied. In this regime one has to consider the case of spin- $\frac{1}{2}$ electrons.

In the presence of electron spins the Hamiltonian (23a) - (23d) has to be modified to account for the two species of electrons: spin- $\uparrow$ and spin- $\downarrow$. Each of the spin subsystems can be bosonized independently, and the Hamiltonian takes the form 13

$$
\begin{aligned}
H_{R}^{(0)} & =\frac{v_{F}}{2 \pi} \sum_{\sigma=\uparrow, \downarrow} \int_{-\infty}^{\infty}\left\{\pi^{2} \Pi_{\sigma}^{2}(x)+\left[\partial_{x} \phi_{\sigma}(x)\right]^{2}\right\} d x, \\
H_{R}^{\prime} & =-\frac{D}{\pi}|r|\left\{\cos \left[2 \phi_{\uparrow}(0)\right]+\cos \left[2 \phi_{\downarrow}(0)\right]\right\} \\
H_{C} & =E_{C}\left\{\hat{n}+\frac{1}{\pi}\left[\phi_{\uparrow}(0)+\phi_{\downarrow}(0)\right]-N\right\}^{2} .
\end{aligned}
$$

To find the thermoelectric coefficient (22) one has to find the correlator $K(\tau)$. Similarly to the case of spinless electrons, $K(\tau)$ can be expressed in terms of the imaginarytime functional integral (25), where

$$
Z(\tau)=\int \exp \left[-\mathcal{S}_{0}-\mathcal{S}_{C}(\tau)-\mathcal{S}^{\prime}\right] \mathcal{D} \phi_{c} \mathcal{D} \phi_{s} .
$$

Here we have introduced the charge and spin fields $\phi_{c, s}(x, t)=\left[\phi_{\uparrow}(x, t) \pm \phi_{\downarrow}(x, t)\right] / \sqrt{2}$. The action in Eq. (37) is expressed in terms of these variables as

$$
\begin{aligned}
& \mathcal{S}_{0}=\sum_{\alpha=c, s} \int_{0}^{\beta} d t \int d x \frac{v_{F}}{2 \pi}\left[\frac{\left(\partial_{t} \phi_{\alpha}\right)^{2}}{v_{F}^{2}}+\left(\partial_{x} \phi_{\alpha}\right)^{2}\right] \\
& \mathcal{S}_{C}(\tau)=\int_{0}^{\beta} d t E_{C}\left[n_{\tau}(t)+\frac{\sqrt{2}}{\pi} \phi_{c}(0, t)-N\right]^{2}, \\
& \mathcal{S}^{\prime}=-\int_{0}^{\beta} d t \frac{2 D}{\pi}|r| \cos \left[\sqrt{2} \phi_{c}(0, t)\right] \cos \left[\sqrt{2} \phi_{s}(0, t)\right] .
\end{aligned}
$$

Similar to the case of spinless electrons, in the absence of backscattering in the constriction the calculation of $K(\tau)$ reduces to evaluation of a gaussian functional integral (37). Clearly, at $r=0$ the integral over $\phi_{s}$ is unaffected by $n_{\tau}(t)$; therefore the integrals over the spin degrees of freedom in the numerator and denominator of Eq. 25 cancel. One can easily see that the action (38a) and (38b) of the charge mode is identical to that of the spinless problem (27a) and (27b) upon the substitution $E_{C} \rightarrow 2 E_{C}, n_{\tau}(t) \rightarrow n_{\tau}(t) / \sqrt{2}$, and $N \rightarrow N / \sqrt{2}$. Making the respective modifications to the derivation of $K_{0}(\tau)$ in Appendix B 1, we find

$$
K_{c}(\tau)=\frac{\pi^{2} T}{2 \gamma E_{C}} \frac{1}{|\sin (\pi T \tau)|}
$$

Substituting the analytic continuation of this result to $\tau=1 / 2 T+i t$ into Eq. (22), we find $G_{T}=0$. As it was explained in Sec. III C, this is the consequence of the fact that the system possesses electron-hole symmetry at $r=0$.

The rest of this section is organized as follows. In Sec. III D 1 we calculate the thermopower within the second-order perturbation theory in the reflection amplitude $r$. We show that the perturbative result diverges at low temperatures. We then find the thermopower at arbitrarily low temperatures in Sec. III D 2 using a nonperturbative approach.

\section{Perturbation theory}

At non-vanishing backscattering the correction to $K_{c}(\tau)$ appears in the second order in $r$. Indeed, the firstorder correction can be expressed in the form (29). It is easy to check that unlike the case of spinless electrons, the average $\left\langle\mathcal{S}^{\prime}\right\rangle$ vanishes, because the fluctuations of the spin mode $\phi_{s}(0, t)$ are not suppressed at low frequencies by the charging energy term (38b). Expanding Eqs. (25) and (37) to second order in $r$, we find

$$
K(\tau)=K_{c}(\tau)\left[1+\frac{1}{2}\left(\left\langle\mathcal{S}^{\prime 2}\right\rangle_{\tau}-\left\langle\mathcal{S}^{\prime 2}\right\rangle_{0}\right)\right]
$$

Similarly to Eq. (30), the averaging $\langle\ldots\rangle_{\tau}$ here is performed with the action $\mathcal{S}_{0}+\mathcal{S}_{C}(\tau)$ given by (38a) and $(38 \mathrm{~b})$. Using the explicit form (38c) of $\mathcal{S}^{\prime}$, we get

$$
\left\langle\mathcal{S}^{\prime 2}\right\rangle_{\tau}=\frac{4 D^{2}|r|^{2}}{\pi^{2}} \int_{0}^{\beta} \int_{0}^{\beta} \kappa_{c}\left(t, t^{\prime} ; \tau\right) \kappa_{s}\left(t, t^{\prime}\right) d t d t^{\prime},
$$

where we have introduced the correlators

$$
\begin{aligned}
\kappa_{c}\left(t, t^{\prime} ; \tau\right) & =\left\langle\cos \left[\sqrt{2} \phi_{c}(0, t)\right] \cos \left[\sqrt{2} \phi_{c}\left(0, t^{\prime}\right)\right]\right\rangle_{\tau}, \\
\kappa_{s}\left(t, t^{\prime}\right) & =\left\langle\cos \left[\sqrt{2} \phi_{s}(0, t)\right] \cos \left[\sqrt{2} \phi_{s}\left(0, t^{\prime}\right)\right]\right\rangle_{0} .
\end{aligned}
$$

The spin fluctuations are completely decoupled from the charging action (38b), rendering the correlator $\kappa_{s}$ independent of $\tau$. The calculation of the correlators $\kappa_{c}$ and $\kappa_{s}$ reduces to evaluation of gaussian integrals. In Appendix $\mathrm{C} 1$ we find 


$$
\begin{aligned}
\kappa_{c}\left(t, t^{\prime} ; \tau\right)=\frac{\gamma E_{C}}{\pi D} \operatorname{Re}\left(e^{2 i \pi N} e^{-i\left[\chi_{\tau}(t)+\chi_{\tau}\left(t^{\prime}\right)\right]}\right. \\
\left.+e^{-i\left[\chi_{\tau}(t)-\chi_{\tau}\left(t^{\prime}\right)\right]}\right), \\
\kappa_{s}\left(t, t^{\prime}\right)=\frac{\pi T}{2 D} \frac{1}{\left|\sin \left[\pi T\left(t-t^{\prime}\right)\right]\right|}
\end{aligned}
$$

where we have introduced the notation

$$
\begin{aligned}
\chi_{\tau}(t) & =\pi n_{\tau}(t)+\delta \chi_{\tau}(t), \\
\delta \chi_{\tau}(t) & =\sum_{n=1}^{\infty} \frac{\sin [2 \pi T(t-\tau) n]-\sin [2 \pi T t n]}{n+\frac{E_{C}}{\pi^{2} T}} .
\end{aligned}
$$

In Eq. (43) we have assumed $\left|t-t^{\prime}\right| \gg E_{C}^{-1}$; we will see that this region gives the leading contribution to the integral (41).

As discussed in Sec. III , only the odd in $N$ part (33) of the correlator $K(\tau)$ contributes to the thermoelectric coefficient (22). Keeping only the odd part of Eq. (43), from $(40)$ in the second order in $r$ we find

$$
\begin{aligned}
K_{\text {odd }}(\tau) & =K_{c}(\tau) \frac{2 \gamma E_{C} T}{\pi^{2}}|r|^{2} \sin (2 \pi N) \mathcal{I}(\tau), \\
\mathcal{I}(\tau) & =\int_{0}^{\beta} d t \sin \chi_{\tau}(t) \int_{0}^{\beta} \frac{\cos \chi_{\tau}\left(t^{\prime}\right)}{\left|\sin \left[\pi T\left(t-t^{\prime}\right)\right]\right|} d t^{\prime} .
\end{aligned}
$$

In evaluating the integral $\mathcal{I}(\tau)$ one should keep in mind that the denominator in Eq. (47) is written for $\left|t-t^{\prime}\right| \gg$ $E_{C}^{-1}$. Thus the logarithmic divergence at $t=t^{\prime}$ should be cut off at $\left|t-t^{\prime}\right| \sim E_{C}^{-1}$.

To evaluate the integral $\mathcal{I}(\tau)$ we first notice that away from the points $t=0, \tau, \beta$ the correction $\delta \chi_{\tau}(t)$ in Eq. (45) is small in $T / E_{C}$,

$$
\delta \chi_{\tau}(t) \simeq \frac{\pi^{2} T}{2 E_{C}}\{\cot [\pi T(t-\tau)]-\cot [\pi T t]\} .
$$

One can neglect this correction in the argument of the cosine in Eq. (47) and replace $\cos \chi_{\tau}\left(t^{\prime}\right)=\operatorname{sgn}\left(t^{\prime}-\tau\right)$. Then the integral over $t^{\prime}$ can be evaluated with logarithmic accuracy:

$$
\begin{aligned}
\mathcal{I}(\tau) \simeq \frac{2}{\pi T} \int_{0}^{\beta} d t & \sin \chi_{\tau}(t)\left[-\ln \tan \frac{\pi T t}{2}\right. \\
+ & \left.\operatorname{sgn}(t-\tau) \ln \left(\frac{E_{C}}{T} \tan \frac{\pi T|t-\tau|}{2}\right)\right] .
\end{aligned}
$$

To leading order in $T / E_{C}$ one can replace $\sin \chi_{\tau}(t)=$ $\delta \chi_{\tau}(t) \operatorname{sgn}(t-\tau)$. Using the approximation (48) we then obtain with logarithmic accuracy

$$
K_{\text {odd }}(\tau)=-K_{c}(\tau) \frac{8 \gamma}{\pi^{2}}|r|^{2} \sin (2 \pi N) \ln \frac{E_{C}}{T} \ln \tan \frac{\pi T \tau}{2} .
$$

Substituting this result for $K(\tau)$ in Eq. (22) we find

$$
G_{T}=-\frac{8 \pi}{9} \frac{G_{L}}{e} \frac{T}{E_{C}} \ln \frac{E_{C}}{T}|r|^{2} \sin (2 \pi N) .
$$

The second-order perturbation theory result for the thermopower can be found from Eq. (8) using the result $G=G_{L} \frac{\pi^{3} T}{8 \gamma E_{C}}$ of Ref. 14 for the conductance of the device at $r=0$,

$$
S=-\frac{64 \gamma}{9 \pi^{2}} \frac{1}{e} \ln \frac{E_{C}}{T}|r|^{2} \sin (2 \pi N) .
$$

This result applies at $T \ll E_{C}$ and, similarly to the spinless case (35), the thermopower vanishes at $r \rightarrow 0$. It is important to note that unlike the spinless case (35), the thermopower (51) diverges at $T \rightarrow 0$. This means, in particular, that the perturbation theory leading to Eq. (51) fails at sufficiently low temperatures. In the next section we perform a non-perturbative calculation and establish the true behavior of the thermopower at $T \rightarrow 0$.

\section{Non-perturbative treatment}

The logarithmic growth of the thermopower (51) at low temperature indicates that the thermoelectric properties of the system are controlled by the spin and charge fluctuations at frequencies below $E_{C}$. In this section we construct a theory that describes the low-energy properties of the system exactly and enables us to obtain a non-perturbative expression for the thermopower at arbitrarily low temperatures. This derivation was outlined in Ref. 17.

As we already discussed, at $r=0$ the contributions of the spin fluctuations to the functional integrals in the numerator and the denominator of Eq. (25) cancel each other, and the ratio of the functional integrals over the charge degrees of freedom is equal to the correlator $K_{c}(\tau)$, Eq. (39). The effect of small but finite $r$ on the charge modes is negligible, because their fluctuations at low energies are suppressed by the charging energy. However, even a small backscattering $r$ pins the fluctuations of the spin modes and changes their low-frequency dynamics dramatically 14 Therefore one can account for the small backscattering by presenting the correlator (25) in the form

$$
K(\tau)=K_{c}(\tau) K_{s}(\tau), \quad K_{s}(\tau)=\frac{Z_{s}(\tau)}{Z_{s}(0)},
$$

where $Z_{s}(\tau)$ is the functional integral over the slow spin modes, averaged over the fast charge modes.

The calculation of $Z_{s}(\tau)$ amounts to integrating out the fast charge degrees of freedom in the functional integral (37). Since the spin and charge fluctuations are only coupled by the backscattering term $(38 \mathrm{~d})$, this procedure reduces to the averaging of $\cos \left[\sqrt{2} \phi_{c}(0, t)\right]$ with the gaussian action $\mathcal{S}_{0}+\mathcal{S}_{C}(\tau)$. Indeed, one can rewrite Eqs. (42a) and (43) as 


$$
\begin{gathered}
\left\langle\cos \left[\sqrt{2} \phi_{c}(0, t)\right] \cos \left[\sqrt{2} \phi_{c}\left(0, t^{\prime}\right)\right]\right\rangle_{\tau}=\Upsilon_{\tau}(t) \Upsilon_{\tau}\left(t^{\prime}\right), \\
\Upsilon_{\tau}(t)=\sqrt{\frac{2 \gamma E_{C}}{\pi D}} \cos \left[\chi_{\tau}(t)-\pi N\right] .
\end{gathered}
$$

One can see from Eq. (53) that at $\left|t-t^{\prime}\right| \gg E_{C}^{-1}$ the correlator (42a) factorizes into the product of the averages of the cosines, and that $\left\langle\cos \left[\sqrt{2} \phi_{c}(0, t)\right]\right\rangle_{\tau}=\Upsilon_{\tau}(t)$. It is clear that the higher-point correlators will also factorize into the product of averages. One can therefore simply replace the $\cos \left[\sqrt{2} \phi_{c}(0, t)\right]$ in the action (38d) by $\Upsilon_{\tau}(t)$ and obtain the effective action for the spin degrees of freedom in the form

$$
\begin{aligned}
\mathcal{S}_{\tau}= & \int_{0}^{\beta} d t \int d x \frac{v_{F}}{2 \pi}\left[\frac{\left(\partial_{t} \phi_{s}\right)^{2}}{v_{F}^{2}}+\left(\partial_{x} \phi_{s}\right)^{2}\right] \\
& -\int_{0}^{\beta} \sqrt{\frac{4 D}{v_{F}}} \lambda_{\tau}(t) \cos \left[\sqrt{2} \phi_{s}(0, t)\right] d t,
\end{aligned}
$$

where we have introduced the notation

$$
\begin{aligned}
\lambda_{\tau}(t) & =\Lambda \cos \left[\chi_{\tau}(t)-\pi N\right] \\
& =\Lambda(-1)^{n_{\tau}(t)} \cos \left[\delta \chi_{\tau}(t)-\pi N\right], \\
\Lambda & =\sqrt{\frac{2 \gamma v_{F} E_{C}}{\pi^{3}}}|r| .
\end{aligned}
$$

The procedure leading to the action (55) implied that all the relevant time scales of the problem are longer than $E_{C}^{-1}$. Therefore one has to integrate out the fluctuations of the spin degrees of freedom with frequencies exceeding $E_{C}$. This procedure is straightforward and amounts to replacing $D$ with the new bandwidth $\sim E_{C}$. Thus Eq. (55) gives the effective action of the problem, provided the bandwidth $D \sim E_{C}$.

One can now find the correlator $K(\tau)$ using Eq. (52) where the functional integral $Z_{s}(\tau)$ is defined as $Z_{s}(\tau)=$ $\int e^{-\mathcal{S}_{\tau}} \mathcal{D} \phi_{s}$. For the subsequent calculations it will be convenient to use the hamiltonian formulation of the problem and express $Z_{s}(\tau)$ as the trace of the timeordered exponential:

$$
Z_{s}(\tau)=\operatorname{Tr}\left\{T_{t} \exp \left[-\int_{0}^{\beta} H_{\tau}(t) d t\right]\right\}
$$

where the time dependent Hamiltonian $H_{\tau}$ is given by

$$
\begin{aligned}
H_{\tau}(t)= & \frac{v_{F}}{2 \pi} \int_{-\infty}^{\infty}\left\{\pi^{2} \Pi_{s}^{2}(x)+\left[\partial_{x} \phi_{s}(x)\right]^{2}\right\} d x \\
& -\sqrt{\frac{4 D}{v_{F}}} \lambda_{\tau}(t) \cos \left[\sqrt{2} \phi_{s}(0)\right] .
\end{aligned}
$$

The small parameter of the problem $r$ enters through $\lambda_{\tau}(t)$. In order to evaluate $K(\tau)$ in all orders in $\lambda_{\tau}$ we refermionize the Hamiltonian (59) following Ref. 13 and find

$$
H_{\tau}(t)=\int_{-\infty}^{\infty}\left[\xi_{k} c_{k}^{\dagger} c_{k}-\lambda_{\tau}(t)\left(c+c^{\dagger}\right)\left(c_{k}-c_{k}^{\dagger}\right)\right] d k .
$$

Here $\xi_{k}=v_{F} k$; the operators $c_{k}^{\dagger}$ and $c_{k}$ satisfying the anticommutation relations $\left\{c_{k}, c_{k^{\prime}}^{\dagger}\right\}=\delta\left(k-k^{\prime}\right)$ create and destroy chiral fermions. Finally, $c$ is a fermion annihilation operator anticommuting with $c_{k}$ and $c_{k}^{\dagger}$.

Although the Hamiltonian (60) is quadratic in the fermion operators, the time dependence of $\lambda_{\tau}(t)$ makes the evaluation of the trace (58) non-trivial. It is clear from Eq. (45b) that at $T / E_{C} \ll 1$ the main time dependence is due to the factor $(-1)^{n_{\tau}(t)}$ in the definition of $\lambda_{\tau}(t)$, Eq. (56). One can greatly simplify the calculation by eliminating this time dependence with the following trick. Note that the unitary transformation with the operator

$$
U=(-1)^{c^{\dagger} c}=\left(c-c^{\dagger}\right)\left(c+c^{\dagger}\right)
$$

changes the sign of $\lambda_{\tau}$ in the Hamiltonian (60). Therefore the factor $(-1)^{n_{\tau}(t)}$ can be accounted for by adding operators $U(\tau)$ and $U(0)$ to the trace (58),

$$
Z_{s}(\tau)=\operatorname{Tr}\left\{T_{t} \exp \left[-\int_{0}^{\beta}\left[H_{0}+H_{\tau}^{\prime}(t)\right] d t\right] U(\tau) U(0)\right\} .
$$

Here $H_{0}+H_{\tau}^{\prime}(t)$ is obtained from the Hamiltonian (60) by replacing $\lambda_{\tau}(t) \rightarrow \lambda_{\tau}(t) /(-1)^{n_{\tau}(t)}$. Its time-independent part $H_{0}$ is given by (60) at $\tau=0$, and the correction is

$$
\begin{aligned}
H_{\tau}^{\prime}(t) & =\Lambda\left\{\cos (\pi N)-\cos \left[\delta \chi_{\tau}(t)-\pi N\right]\right\}\left(c+c^{\dagger}\right) \Psi, \\
\Psi & =\int_{-\infty}^{\infty}\left(c_{k}-c_{k}^{\dagger}\right) d k .
\end{aligned}
$$

The perturbation $H_{\tau}^{\prime}(t)$ vanishes at $T / E_{C} \rightarrow 0$. In this limit the spin contribution to the correlator (52) becomes the Green's function of operators $U$,

$$
K_{s}^{(0)}(\tau)=\left\langle T_{t} U(\tau) U(0)\right\rangle_{0},
$$

where $\langle\ldots\rangle_{0}$ denotes averaging over the equilibrium thermal distribution with the Hamiltonian $H_{0}$. The explicit analytic result for this quantity is given by formula (66) of Ref. 14. The result is an even function of the gate voltage $N$, and therefore within the approximation $H_{\tau}^{\prime}(t)=0$ the thermoelectric coefficient $G_{T}$ vanishes.

To find the leading contribution to $G_{T}$ at small $T / E_{C}$ we expand Eq. (62) to first order of the perturbation theory in $H_{\tau}^{\prime}(t)$. The correction to $K_{s}(\tau)$ has the form

$$
K_{s}^{(1)}(\tau)=-\int_{0}^{\beta} d t\left\langle T_{t} H_{\tau}^{\prime}(t) U(\tau) U(0)\right\rangle_{0} .
$$

This correction is evaluated with logarithmic accuracy in Appendix C2.

$$
\begin{aligned}
K_{s}^{(1)}(\tau)= & -\frac{8 \gamma}{\pi^{2}}|r|^{2} \sin (2 \pi N) \ln \frac{E_{C}}{T+\Gamma} \\
& \times \int_{-\infty}^{\infty} \frac{\xi d \xi}{\xi^{2}+\Gamma^{2}} \frac{e^{\xi \tau}}{e^{\beta \xi}+1}, \\
\Gamma= & \frac{8 \gamma E_{C}}{\pi^{2}}|r|^{2} \cos ^{2}(\pi N) .
\end{aligned}
$$


It is important to note that although this result is the first-order correction in $H_{\tau}^{\prime}(t)$, it is non-perturbative in the reflection amplitude $r$.

Substituting the correlator $K(\tau)$ in the form (52) with $K_{c}$ and $K_{s}$ given by Eqs. (39) and (67) into the expression for the thermoelectric coefficient (22), we obtain

$$
\begin{aligned}
G_{T}= & -\frac{1}{6 \pi} \frac{G_{L}}{e} \frac{T}{E_{C}} \ln \frac{E_{C}}{T+\Gamma}|r|^{2} \sin (2 \pi N) \\
& \times \int_{-\infty}^{\infty} \frac{x^{2}\left(x^{2}+\pi^{2}\right) d x}{\left[x^{2}+(\Gamma / T)^{2}\right] \cosh ^{2}(x / 2)} .
\end{aligned}
$$

At temperatures $T \gg \Gamma$ this expression reproduces the perturbative result (50). The latter is valid until $T \sim \Gamma$, and at $T \ll \Gamma$ the thermoelectric coefficient $G_{T}$ becomes

$$
G_{T}=-\frac{\pi^{7}}{60 \gamma^{2}} \frac{G_{L}}{e} \frac{T^{3}}{E_{C}^{3}} \frac{1}{|r|^{2}} \frac{\sin (\pi N)}{\cos ^{3}(\pi N)} \ln \frac{1}{|r|^{2} \cos ^{2}(\pi N)} .
$$

The dependence of $G_{T}$ on the small reflection amplitude illustrates the non-perturbative nature of this result. It is also worth noting that at low temperatures the dependence of $G_{T}$ on the gate voltage $N$ is strongly nonsinusoidal.

To find the thermopower $S=G_{T} / G$ one can use the expression (69) and the non-perturbative result of Ref. 14 for the conductance $G$ of the SET,

$$
G=\frac{G_{L} \Gamma}{8 \gamma E_{C}} \int_{-\infty}^{\infty} \frac{\left(x^{2}+\pi^{2}\right) d x}{\left[x^{2}+(\Gamma / T)^{2}\right] \cosh ^{2}(x / 2)} .
$$

At relatively high temperatures $T \gg \Gamma$ the thermopower $S=G_{T} / G$ obtained from Eqs. (69) and (71) coincides with the perturbative expression (51). In the more interesting case of low temperatures $T \ll \Gamma$, we find

$$
S=-\frac{\pi^{3}}{5} \frac{1}{e} \frac{T}{E_{C}} \tan (\pi N) \ln \frac{1}{|r|^{2} \cos ^{2}(\pi N)} .
$$

The new energy scale $\Gamma$ arising from the nonperturbative solution is always small compared to the charging energy, see Eq. (68). It is important to keep in mind that $\Gamma$ is a function of the gate voltage, and vanishes near the Coulomb blockade peaks, $N= \pm \frac{1}{2}, \pm \frac{3}{2}, \pm \frac{5}{2}, \ldots$ As a result, even at $T \ll E_{C}|r|^{2}$ the perturbative results (50) and (51) are still valid near the conductance peaks, whereas in the valleys the new asymptotics $(70)$ and (72) apply. The crossover between these asymptotics occurs at the values of $N$ where $\Gamma=T$, i.e., according to Eq. (68) at a distance $\delta N \sim \sqrt{T / E_{C}|r|^{2}}$ from the centers of the conductance peaks. At these points the thermopower reaches its maximum absolute value $S_{\max }$, which can be estimated by substituting $N=\frac{1}{2}+\delta N$ in either Eq. (51) or Eq. (72), resulting in

$$
S_{\max } \sim e^{-1}|r| \sqrt{\frac{T}{E_{C}}} \ln \frac{E_{C}}{T} .
$$

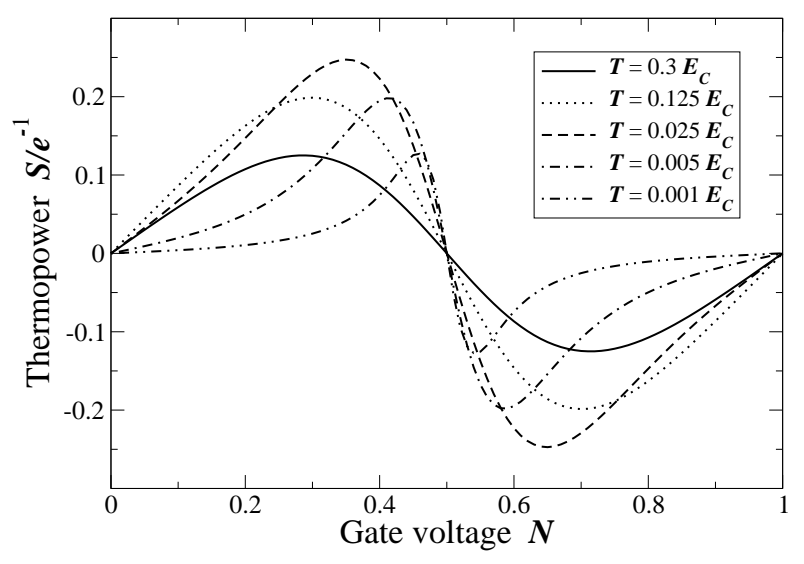

FIG. 2. Thermopower of SET as a function of gate voltage at different temperatures. The curves for $S=G_{T} / G$ are obtained numerically from Eqs. (69) and (71) at $|r|^{2}=0.1$ and $T / E_{C}=0.3,0.125,0.025,0.005,0.001$. As the temperature is lowered, the amplitude of the thermopower oscillations first grows according to Eq. (51) and then decreases in agreement with Eq. (73). Below the crossover temperature $\sim E_{C}|r|^{2}$ the shape of the oscillations becomes non-sinusoidal.

The exact shape of the Coulomb blockade oscillations of the thermopower found from Eqs. (69) and (71) is illustrated in Fig. 2.

\section{SUMMARY AND DISCUSSION}

We presented a theory of the thermopower of single electron transistors in the regime when the coupling of the quantum dot to one of the leads is strong. The theory is applicable to devices with relatively large dots, where the effects of finite quantum level spacing can be neglected, and the main transport mechanism is inelastic cotunneling. Using the fact that the coupling to one of the two leads is weak, we obtained the expression (22) for the thermoelectric coefficient $G_{T}$ in terms of the correlator $K(\tau)$ describing the charging of the dot strongly coupled to the other lead. The general expression (22) is applicable to contacts with arbitrary coupling. We applied it to the case of coupling via a quantum point contact with a single transverse mode and almost perfect transmission, $|r| \ll 1$. In the case of spin-polarized electrons we found sinusoidal Coulomb blockade oscillations of the thermopower with the amplitude $\sim e^{-1}|r| T / E_{C}$, Eq. (35). Experimentally the polarization of electron spins can be achieved by applying a strong magnetic field. In the absence of the magnetic field the thermopower is given by the ratio of non-perturbative expressions (69) and (71). At relatively high temperatures $T \gg E_{C}|r|^{2}$ the Coulomb blockade oscillations of $S$ are sinusoidal, with the amplitude $\sim e^{-1}|r|^{2} \ln \left(E_{C} / T\right)$, 
Eq. (51). At lower temperatures $T \ll E_{C}|r|^{2}$ the oscillations are non-sinusoidal, Fig. 2, and their amplitude is given by Eq. (73).

We are aware of only one experiment on the thermopower of SET in the strong coupling regime, Ref. 10 . In this experiment the Coulomb blockade oscillations of the thermopower $S(N)$ were measured at different values of the reflection coefficient. Only one published curve $S(N)$, measured at $|r|^{2}=0.2 \pm 0.1$ approached the strong tunneling limit $|r| \ll 1$. In this case the thermopower remained sinusoidal even at the lowest available temperatures. To observe the more interesting nonsinusoidal behavior of $S(N)$ one would have to measure the thermopower at lower temperature to reach the regime $T \ll E_{C}|r|^{2}$. This may require making a sample with a larger quantum dot to ensure that the lowest temperature is still large compared to the quantum level spacing.

\section{ACKNOWLEDGMENTS}

This research was supported by the NSF Grants No. DMR-9974435 and DMR-9984002, and by the Sloan and the Packard foundations. We are also grateful to B.L. Altshuler and L. Molenkamp for valuable discussions.

\section{APPENDIX A: TUNNELING DENSITY OF STATES}

In this appendix we present the derivation of Eq. (14) for the tunneling density of states $\nu(\epsilon)$. We start with the standard expression for the density of states

$$
\nu(\epsilon)=\frac{i}{2 \pi}\left[G^{R}(\epsilon)-G^{A}(\epsilon)\right]
$$

where $G^{R}$ and $G^{A}$ are the retarded and advanced Green's functions, which can be obtained by the analytic continuation of the Matsubara Green's function $G\left(\epsilon_{n}\right)$.

In the frequency representation the Matsubara Green's function can be written as

$$
G\left(\epsilon_{n}\right)=\int_{0}^{\beta} d \tau \exp \left(i \epsilon_{n} \tau\right) G(\tau)
$$

where $\epsilon_{n}=\pi T(2 n+1)$ are the fermionic Matsubara frequencies. Depending on the sign of $\epsilon_{n}$ the $\tau$ integration contour can be distorted to the upper or lower half plane as shown in Fig. 3 .

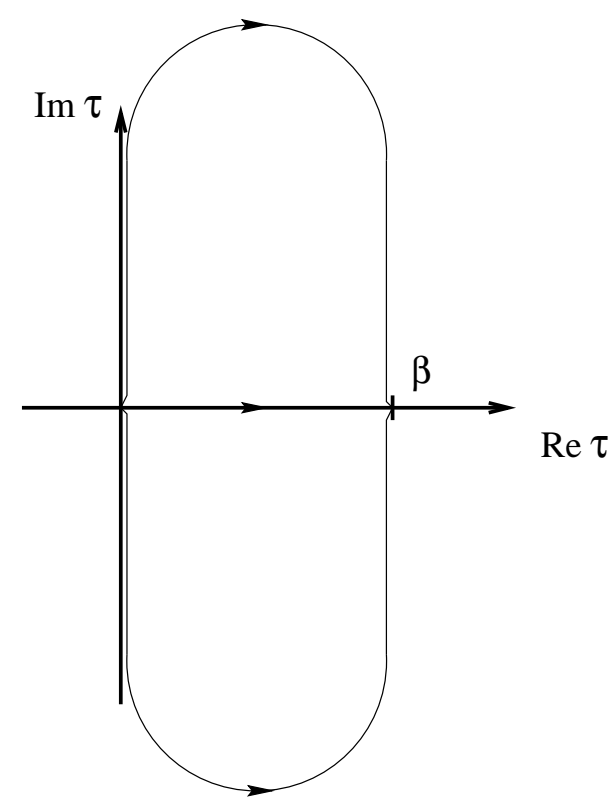

FIG. 3. Representation of the deformation of the $\tau$-integration contour in Eq. A2 . For positive $\epsilon_{n}$ the contour should be distorted into the upper half plane, and for negative $\epsilon_{n}$ — into the lower half plane.

Keeping in mind that the retarded Green's function $G^{R}\left(i \epsilon_{n}\right)=G\left(\epsilon_{n}\right)$ at $\epsilon_{n}>0$, and using the fact that for the fermionic Matsubara frequencies $\exp \left(i \epsilon_{n} \beta\right)=-1$, we can then express the retarded Green function as

$$
\begin{aligned}
G^{R}\left(i \epsilon_{n}\right)=i \int_{0}^{\infty} & d t \exp \left(-\epsilon_{n} t\right) \\
& \times[G(i t+0)+G(\beta-0+i t)] .
\end{aligned}
$$

The analytic continuation to real frequencies can now be performed in the last line of Eq. (A3) through the substitution $\epsilon_{n} \rightarrow-i \epsilon$.

We then obtain a similar expression for the advanced Green's function $G^{A}(\epsilon)$ using the relation $G^{A}\left(i \epsilon_{n}\right)=$ $G\left(\epsilon_{n}\right)$ at $\epsilon_{n}<0$. Combining the two results, we find the following expression for the density of states (A1)

$$
\begin{aligned}
\nu(\epsilon)= & -\frac{1}{2 \pi} \int_{-\infty}^{\infty} d t \exp (i \epsilon t) G(i t+0) \\
& -\frac{1}{2 \pi} \int_{-\infty}^{\infty} d t \exp (i \epsilon t) G(\beta-0+i t) .
\end{aligned}
$$

Since the Green's function $G(\tau)$ is analytic everywhere except on the lines $\operatorname{Re} \tau=0, \pm \beta, \pm 2 \beta, \ldots$, we can shift the integration contour in the first line of Eq. (A4) by $t \rightarrow t-i \beta / 2$ and in the second one by $t \rightarrow t+i \beta / 2$. As a result we obtain Eq. (14).

\section{APPENDIX B: $K(\tau)$ FOR SPINLESS ELECTRONS}

In this appendix we derive the results (28) and (31) for the correlator $K(\tau)$ in the spinless case. 


\section{Evaluation of $K_{0}(\tau)$, Eq. (28)}

To derive Eq. (28) we evaluate gaussian integral 26) under the assumption $\mathcal{S}^{\prime}=0$. First, we find $\phi_{\tau}(x, t)$ that minimizes the action $\mathcal{S}_{0}+\mathcal{S}_{C}(\tau)$. Differentiating Eqs. (27a and (27b) with respect to $\phi(x, t)$, we find

$$
\partial_{t}^{2} \phi_{\tau}+v_{F}^{2} \partial_{x}^{2} \phi_{\tau}-2 v_{F} E_{C}\left[n_{\tau}(t)+\frac{1}{\pi} \phi_{\tau}-N\right] \delta(x)=0 .
$$

The solution of this equation has the form

$$
\phi_{\tau}(x, t)=\pi N-T \sum_{\omega_{n}} \frac{E_{C} \exp \left(-\frac{\left|\omega_{n} x\right|}{v_{F}}\right)}{\left|\omega_{n}\right|+\frac{E_{C}}{\pi}} n_{\tau}\left(\omega_{n}\right) e^{-i \omega_{n} t},
$$

where $\omega_{n}=2 \pi n T$ are bosonic Matsubara frequencies, and $n_{\tau}\left(\omega_{n}\right)$ is the Fourier transform of $n_{\tau}(t)$, Eq. (24),

$$
n_{\tau}\left(\omega_{n}\right)=\frac{e^{i \omega_{n} \tau}-1}{i \omega_{n}}
$$

In the calculation of the correlator $K_{0}(\tau)$ the integrals over the fluctuations of the field $\phi(x, t)$ about the saddle points $\phi_{\tau}(x, t)$ and $\phi_{0}(x, t)$ in the numerator and the denominator of Eq. (25) cancel each other. Thus $K_{0}(\tau)$ is given by the ratio of the saddle-point values of the respective integrals. Substituting (B1) into (27a) and (27b) we find the saddle point action in the form

$$
\left[\mathcal{S}_{0}+\mathcal{S}_{C}(\tau)\right]_{\phi=\phi_{\tau}(x, t)}=\frac{E_{C}}{\pi^{2} T} \sum_{n=1}^{\infty} \frac{1-\cos (2 \pi T \tau n)}{n\left(n+\frac{E_{C}}{2 \pi^{2} T}\right)} .
$$

In the denominator of Eq. (25) we have the saddle point action at $\tau=0$; according to Eq. (B3) it vanishes. In the numerator of Eq. (25) the time $\tau$ is finite. Assuming $\tau \gg E_{C}^{-1}$ and $T \ll E_{C}$, we find

$$
-\left[\mathcal{S}_{0}+\mathcal{S}_{C}(\tau)\right]_{\phi=\phi_{\tau}(x, t)} \simeq 2 \ln \frac{\pi^{2} T}{\gamma E_{C}|\sin (\pi T \tau)|} .
$$

The correlator $K_{0}(\tau)$ is now found by exponentiation of Eq. (B4). The result is given by Eq. (28).

\section{Evaluation of $K(\tau)$ to first order in $r$, Eq. (31)}

To derive the first-order correction (31) to the correlator $K_{0}(\tau)$ one has to evaluate the gaussian functional integral (30). It is convenient to integrate with respect to fluctuations $\varphi=\phi-\phi_{\tau}$ of the field $\phi(x, t)$ about the saddle point $\phi_{\tau}(x, t)$. Then the integral (30) takes the form

$$
\left\langle\mathcal{S}^{\prime}\right\rangle_{\tau}=\operatorname{Re} \int_{0}^{\beta} d t e^{2 i \phi_{\tau}(0, t)}\left[-\frac{D}{\pi}|r|\left\langle e^{2 i \varphi(0, t)}\right\rangle\right],
$$

where the averaging $\langle\ldots\rangle$ is performed over the fluctuations around the saddle point $\phi_{\tau}$. This averaging can be viewed as integral (30) with $n_{\tau}$ and $N$ in the charging action $\mathcal{S}_{C}$ set to zero. The evaluation of this integral is straightforward, but lengthy. It can be avoided by noticing that the expression in the square brackets in Eq. (B5) is time-independent and has the meaning of the first-order correction to the ground state energy of the Hamiltonian (23a) $-(23 \mathrm{c})$ at $\hat{n}=N=0$. Substituting its value found in Ref. 13, we get

$$
\left\langle\mathcal{S}^{\prime}\right\rangle_{\tau}=-\frac{\gamma}{\pi^{2}}|r| E_{C} \operatorname{Re} \int_{0}^{\beta} d t e^{2 i \phi_{\tau}(0, t)}
$$

Using Eqs. (B1) and (B2) we now find

$$
\begin{aligned}
\left\langle\mathcal{S}^{\prime}\right\rangle_{\tau}-\left\langle\mathcal{S}^{\prime}\right\rangle_{0}= & -\frac{\gamma}{\pi^{2}}|r| E_{C} \operatorname{Re} e^{2 i \pi N} \\
& \times \int_{0}^{\beta} d t\left(e^{i[F(t)-F(t-\tau)]}-1\right),
\end{aligned}
$$

where

$$
F(t)=2 \sum_{n=1}^{\infty} \frac{\sin (2 \pi T t n)}{n+\frac{E_{C}}{2 \pi^{2} T}}
$$

At $T \ll E_{C}$ the series can be evaluated explicitly for arbitrary $t$,

$$
F(t)= \begin{cases}\frac{2 \pi^{2} T}{E_{C}} \cot (\pi T t), & t \gg E_{C}^{-1}, \\ 2 \int_{0}^{\infty} d y \frac{\sin \left(E_{C} t y / \pi\right)}{1+y}, & t \ll T^{-1} .\end{cases}
$$

In order to find the thermoelectric coefficient (22) we need to find $K(\tau)$ at $\tau \sim T^{-1} \gg E_{C}^{-1}$. At these time scales the details of the short-time behavior of $e^{i F(t)}$ are irrelevant, and one can replace

$$
e^{i F(t)} \rightarrow 1-\frac{\pi^{2} \xi}{E_{C}} \delta(t)+i \sin F(t),
$$

where the constant $\xi \approx 1.59$ is defined as

$$
\xi=\frac{2}{\pi} \int_{0}^{\infty} d x\left[1-\cos \left(2 \int_{0}^{\infty} d y \frac{\sin (x y)}{1+y}\right)\right] .
$$

Substituting the approximation (B10) into the integral in Eq. (B7) and using Eq. (B9), we find

$$
\begin{aligned}
\left\langle\mathcal{S}^{\prime}\right\rangle_{\tau}-\left\langle\mathcal{S}^{\prime}\right\rangle_{0}= & 2 \gamma \xi|r|[\cos (2 \pi N) \\
& \left.-\frac{2 \pi^{2} T}{E_{C}} \sin (2 \pi N) \cot (\pi T \tau)\right] .
\end{aligned}
$$

The calculation of the conductance $G$ and the thermoelectric coefficient $G_{T}$ requires the knowledge of the even and odd in $\tau$ components of $K(\tau)$, respectively. In Eq. (B12) we retained only the leading-order terms in $T / E_{C}$ for each of these components. Substituting Eq. (B12) into (29), we arrive at Eq. (31). 


\section{APPENDIX C: $K(\tau)$ FOR ELECTRONS WITH SPIN}

1. Evaluation of $\kappa_{c}\left(t, t^{\prime} ; \tau\right)$ and $\kappa_{s}\left(t, t^{\prime}\right)$, Eq. (42)

The correlator $\kappa_{c}\left(t, t^{\prime} ; \tau\right)$ defined by Eq. (42a) can be presented in the form

$$
\kappa_{c}\left(t, t^{\prime} ; \tau\right)=\frac{1}{2} \operatorname{Re}\left[\kappa_{c}^{+}\left(t, t^{\prime} ; \tau\right)+\kappa_{c}^{-}\left(t, t^{\prime} ; \tau\right)\right]
$$

where

$$
\kappa_{c}^{ \pm}\left(t, t^{\prime} ; \tau\right)=\left\langle e^{i \sqrt{2}\left[\phi_{c}(0, t) \pm \phi_{c}\left(0, t^{\prime}\right)\right]}\right\rangle_{\tau} .
$$

The calculation of the correlators $\kappa_{\tau}^{ \pm}$amounts to evaluation of gaussian integrals. Similarly to the calculations of Appendix B2, it is convenient to integrate over the fluctuations $\varphi_{c}$ about the saddle point

$$
\phi_{c, \tau}(0, t)=\frac{\pi N}{\sqrt{2}}-T \sum_{\omega_{n}} \frac{\sqrt{2} E_{C}}{\left|\omega_{n}\right|+\frac{2 E_{C}}{\pi}} n_{\tau}\left(\omega_{n}\right) e^{-i \omega_{n} t},
$$

where $n\left(\omega_{n}\right)$ is given by Eq. (B2). The saddle point Eq. (C3) is easily obtained from (B1) by replacing $E_{C} \rightarrow$ $2 E_{C}, n_{\tau}(t) \rightarrow n_{\tau}(t) / \sqrt{2}$, and $N \rightarrow N / \sqrt{2}$. Substituting $\phi_{c}(0, t)=\phi_{c, \tau}(0, t)+\varphi(t)$ into Eq. (C2) we find

$$
\begin{aligned}
\kappa_{c}^{ \pm}\left(t, t^{\prime} ; \tau\right)= & \exp \left\{i \sqrt{2}\left[\phi_{c, \tau}(0, t) \pm \phi_{c, \tau}\left(0, t^{\prime}\right)\right]\right\} \\
& \times \exp \left\{-2\left\langle\varphi_{c}(t)\left[\varphi_{c}(t) \pm \varphi_{c}\left(t^{\prime}\right)\right]\right\rangle\right\} .
\end{aligned}
$$

To evaluate the last factor in Eq. (C4) we introduce the generating functional

$$
W\left[\left\{J\left(\omega_{n}\right)\right\}\right]=\left\langle\exp \left[-T \sum_{\omega_{n}} J\left(\omega_{n}\right) \varphi_{c}\left(-\omega_{n}\right)\right]\right\rangle .
$$

This gaussian integral is completely determined by the saddle point value $\varphi_{c}^{J}(t)$ of the field $\varphi_{c}$,

$$
W\left[\left\{J\left(\omega_{n}\right)\right\}\right]=\exp \left[-\frac{1}{2} T \sum_{\omega_{n}} J\left(\omega_{n}\right) \varphi_{c}^{J}\left(-\omega_{n}\right)\right] .
$$

Next we note that fluctuations of $\varphi_{c}(t)$ coincide with those of $\phi_{c}(0, t)$ at $N=n_{\tau}=0$. Then $n_{\tau}(t)$ in Eq. (38b) plays the role of a source term similar to $J(t)$. More precisely, they are related according to $J(t)=\left(2 \sqrt{2} E_{C} / \pi\right) n_{\tau}(t)$. Then the saddle point $\varphi_{c}^{J}(t)$ can be determined from (C3) at $N=0$ and $n_{\tau}\left(\omega_{n}\right)=$ $\left(\pi / 2 \sqrt{2} E_{C}\right) J\left(\omega_{n}\right)$, and we obtain

$$
W\left[\left\{J\left(\omega_{n}\right)\right\}\right]=\exp \left[\frac{\pi}{4} T \sum_{\omega_{n}} \frac{J\left(\omega_{n}\right) J\left(-\omega_{n}\right)}{\left|\omega_{n}\right|+\frac{2 E_{C}}{\pi}}\right] .
$$

Differentiating the functional $W$ with respect to $J\left(\omega_{n}\right)$ and $J\left(-\omega_{m}\right)$, from Eqs. (C5) and (C6) we find

$$
\left\langle\varphi_{c}\left(-\omega_{n}\right) \varphi_{c}\left(\omega_{m}\right)\right\rangle=\frac{\pi}{2 T} \frac{1}{\left|\omega_{n}\right|+\frac{2 E_{C}}{\pi}} \delta_{n, m} .
$$

In the time representation this result takes the form

$$
\left\langle\varphi_{c}(t) \varphi_{c}\left(t^{\prime}\right)\right\rangle=\frac{\pi}{2} T \sum_{\omega_{n}} \frac{e^{i \omega_{n}\left(t-t^{\prime}\right)}}{\left|\omega_{n}\right|+\frac{2 E_{C}}{\pi}} e^{-\left|\omega_{n}\right| / D} .
$$

The asymptotic behavior of this correlator is

$$
\left\langle\varphi_{c}(t) \varphi_{c}\left(t^{\prime}\right)\right\rangle= \begin{cases}\frac{1}{2} \ln \frac{\pi D}{2 \gamma E_{C} \sqrt{1+\left[D\left(t-t^{\prime}\right)\right]^{2}}}, & \left|t-t^{\prime}\right| \ll \frac{1}{E_{C}}, \\ \frac{\pi^{4} T^{2}}{8 E_{C}^{2} \sin ^{2} \pi T\left(t-t^{\prime}\right)}, & \left|t-t^{\prime}\right| \gg \frac{1}{E_{C}} .\end{cases}
$$

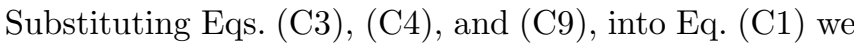
find the correlator $\kappa_{c}\left(t, t^{\prime} ; \tau\right)$ at $\left|t-t^{\prime}\right| \gg E_{C}^{-1}$ in the form (43), where

$$
\chi_{\tau}(t)=\frac{E_{C}}{2 \pi^{2} T} \sum_{n=-\infty}^{\infty} \frac{e^{-i 2 \pi T(t-\tau) n}-e^{-i 2 \pi T t n}}{i n\left(|n|+\frac{E_{C}}{\pi^{2} T}\right)} .
$$

This definition of $\chi_{\tau}(t)$ can be rewritten in the form (45).

Our derivation of $\kappa_{c}\left(t, t^{\prime} ; \tau\right)$ allows one to find $\kappa_{s}\left(t, t^{\prime}\right)$ as well. Indeed, at $E_{C} \rightarrow 0$ the actions of the charge and spin modes are identical. Taking the limit $E_{C} \rightarrow 0$ in Eqs. $(\mathrm{C} 3)$ and $(\overline{\mathrm{C} 8})$, from $(\mathrm{C} 4)$ and $(\overline{\mathrm{C} 1})$ we find the correlator $\kappa_{s}\left(t, t^{\prime}\right)$ at $\left|t-t^{\prime}\right| \gg D^{-1}$ in the form (44).

\section{Evaluation of the correlator $K_{s}^{(1)}(\tau)$, Eq. (67)}

In this Appendix we outline the derivation of the correlator (67) starting from Eq. (66). At small temperature $T \ll E_{C}$ one can expand the expression (63) for $H_{\tau}^{\prime}(t)$ to first order in $\delta \chi_{\tau}$ and present Eq. (66) in the form

$$
\begin{aligned}
K_{s}^{(1)}(\tau) & =\Lambda \sin (\pi N) \int_{0}^{\beta} \delta \chi_{\tau}(t) \Phi(\tau, t) d t, \\
\Phi(\tau, t) & =\left\langle T_{t}\left(c+c^{\dagger}\right)_{t} \Psi(t) U(\tau) U(0)\right\rangle_{0} .
\end{aligned}
$$

Here we introduced the shorthand notation $\left(c+c^{\dagger}\right)_{t} \equiv$ $c(t)+c^{\dagger}(t)$. To evaluate $\Phi(\tau, t)$ we substitute the expression (61) for $U$. Since the operator $\left(c-c^{\dagger}\right)$ commutes with the Hamiltonian $H_{0}$, the Green's function $\left\langle T_{t}\left(c-c^{\dagger}\right)_{\tau}\left(c-c^{\dagger}\right)_{0}\right\rangle_{0}=-1$, and we find

$$
\Phi(\tau, t)=\left\langle T_{t}\left(c+c^{\dagger}\right)_{t} \Psi(t)\left(c+c^{\dagger}\right)_{\tau}\left(c+c^{\dagger}\right)_{0}\right\rangle_{0} .
$$

Considering that the Hamiltonian $H_{0}$ is quadratic in fermion operators, one can use Wick's theorem and present $\Phi(\tau, t)$ in terms of the single particle Green's functions:

$$
\begin{aligned}
\Phi(\tau, t)= & G_{1}(\tau) G_{2}(0)+G_{1}(t-\tau) G_{2}(-t) \\
& -G_{1}(t) G_{2}(\tau-t) .
\end{aligned}
$$


Here $G_{1}(t)$ and $G_{2}(t)$ are defined as

$$
\begin{aligned}
& G_{1}(t)=\left\langle T_{t}\left(c+c^{\dagger}\right)_{t}\left(c+c^{\dagger}\right)_{0}\right\rangle_{0}, \\
& G_{2}(t)=\left\langle T_{t}\left(c+c^{\dagger}\right)_{t} \Psi(0)\right\rangle_{0} .
\end{aligned}
$$

Evaluation of the Green's functions (C14) can be facilitated by noticing that upon the substitution $\Lambda \cos (\pi N)=$ $\lambda$ the Hamiltonian $H_{0}$ coincides with the Hamiltonian

$$
H=\int_{-\infty}^{\infty}\left[\xi_{k} c_{k}^{\dagger} c_{k}-\lambda\left(c+c^{\dagger}\right)\left(c_{k}-c_{k}^{\dagger}\right)\right] d k
$$

in Eq. (44) of Ref. 13. This Hamiltonian was diagonalized to the form 3

$$
H=E+\int_{0}^{\infty} \xi_{k}\left(C_{k}^{\dagger} C_{k}+\tilde{C}_{k}^{\dagger} \tilde{C}_{k}\right) d k
$$

where $E$ is the ground state energy of the Hamiltonian $H$ and the fermion operators $C_{k}$ and $\tilde{C}_{k}$ are given by

$$
\begin{aligned}
C_{k}= & \frac{\xi_{k}}{\sqrt{\xi_{k}^{2}+\Gamma^{2}}} \frac{c_{k}-c_{-k}^{\dagger}}{\sqrt{2}}-\frac{\sqrt{2} \lambda}{\sqrt{\xi_{k}^{2}+\Gamma^{2}}}\left(c+c^{\dagger}\right) \\
& +\frac{\Gamma}{\pi \sqrt{\xi_{k}^{2}+\Gamma^{2}}} \int_{-\infty}^{\infty} \frac{d \xi_{k^{\prime}}}{\xi_{k}-\xi_{k^{\prime}}} \frac{c_{k^{\prime}}-c_{-k^{\prime}}^{\dagger}}{\sqrt{2}}, \\
\tilde{C}_{k}= & \left(c_{k}+c_{-k}^{\dagger}\right) / \sqrt{2} .
\end{aligned}
$$

Here $\Gamma=4 \pi \lambda^{2} / v_{F}$, which in our notations becomes Eq. (68).

To find the Green's functions (C14) we invert the transformation (C17) and obtain

$$
\begin{aligned}
\frac{c_{k}-c_{-k}^{\dagger}}{\sqrt{2}}= & -\frac{\Gamma}{\pi} \int_{-\infty}^{\infty} \frac{d \xi_{k^{\prime}}}{\xi_{k}-\xi_{k^{\prime}}} \frac{\theta\left(\xi_{k^{\prime}}\right) C_{k^{\prime}}+\theta\left(-\xi_{k^{\prime}}\right) C_{-k^{\prime}}^{\dagger}}{\sqrt{\xi_{k^{\prime}}^{2}+\Gamma^{2}}} \\
& +\frac{\xi_{k}}{\sqrt{\xi_{k}^{2}+\Gamma^{2}}}\left[\theta\left(\xi_{k}\right) C_{k}+\theta\left(-\xi_{k}\right) C_{-k}^{\dagger}\right], \quad(\mathrm{C} 19) \\
c+c^{\dagger}= & -2^{3 / 2} \lambda \int_{0}^{\infty} \frac{d k}{\sqrt{\xi_{k}^{2}+\Gamma^{2}}}\left(C_{k}+C_{k}^{\dagger}\right) . \quad(\mathrm{C} 20)
\end{aligned}
$$

Using these results, the definition of $\Psi$, Eq. 64 , and the form (C16) of the Hamiltonian, we easily obtain the Green's functions

$$
\begin{aligned}
& G_{1}(t)=\frac{2 \Gamma}{\pi} \operatorname{sgn} t \int_{-\infty}^{\infty} \frac{d \xi}{\xi^{2}+\Gamma^{2}} \frac{e^{\xi|t|}}{e^{\beta \xi}+1}, \\
& G_{2}(t)=-\frac{4 \lambda}{v_{F}} \int_{-\infty}^{\infty} \frac{\xi d \xi}{\xi^{2}+\Gamma^{2}} \frac{e^{\xi|t|}}{e^{\beta \xi}+1} .
\end{aligned}
$$

The Green's functions $G_{1}(t)$ and $G_{2}(t)$ are odd and even functions of $t$, respectively. Noticing also that $\delta \chi_{\tau}(t)$ given by Eq. (45b) is invariant with respect to the change of variables $t \rightarrow \tau-t$, we conclude that the contributions of the second and third terms in Eq. (C13) to the integral (C10) are equal to each other. Finally, the first term in Eq. (C13) does not contribute to (C10) because it is independent of $t$, and the time integral of $\delta \chi_{\tau}(t)$ vanishes. Therefore we rewrite Eq. (C10) as

$$
K_{s}^{(1)}(\tau)=-2 \Lambda \sin (\pi N) \int_{0}^{\beta} \delta \chi_{\tau}(t) G_{1}(t) G_{2}(\tau-t) d t
$$

Without loss of generality we can assume $\Gamma \sim T \ll E_{C}$. Since $\delta \chi_{\tau}(t) \simeq-\left(\pi^{2} T / 2 E_{C}\right) \cot [\pi T t]$ near $t=0, \beta$, see Eq. (48), the integral (C23) diverges logarithmically at $t \rightarrow 0$ and $t \rightarrow \beta$. These divergences are cut off at the short time scale $E_{C}^{-1}$ and the long time scale $\min \left\{\Gamma^{-1}, T^{-1}\right\}$. Due to the fact that $G_{1}(+0) G_{2}(\tau)=$ $-G_{1}(\beta-0) G_{2}(\tau-\beta)$, the two divergences add up. Therefore with logarithmic accuracy the correlator $\mathrm{C} 23$ is given by

$$
K_{s}^{(1)}(\tau)=\frac{2 \pi \Lambda}{E_{C}} \sin (\pi N) G_{1}(+0) G_{2}(\tau) \ln \frac{E_{C}}{T+\Gamma} .
$$

Substituting $G_{1}(+0)=1$ and the expression $(\mathrm{C} 22)$ for $G_{2}$, we arrive at Eq. 67).

${ }^{1}$ J.M. Ziman, Principles of the Theory of Solids (Cambridge University Press, Cambridge, 1972).

2 A.A. Abrikosov, Fundamentals of the Theory of Metals (North-Holland, Amsterdam, 1988).

3 A.V. Anisovich, B.L. Altshuler, A.G. Aronov, and A.Yu. Zyuzin, JETP Lett. 45, 295 (1987).

${ }^{4}$ G.B. Lesovik and D.E. Khmel'nitskii, Sov. Phys. JETP 67, 957 (1988).

${ }^{5}$ B.Z. Spivak and A.Yu. Zyuzin, Europhys. Lett. 8, 669 (1989).

${ }^{6}$ M. Nahum, T.M. Eiles, and J.M. Martinis, Appl. Phys. Lett. 65, 3123 (1994).

${ }^{7}$ C.W.J. Beenakker, and A.A.M. Staring, Phys. Rev. B 46, 9667 (1992).

${ }^{8}$ A.A.M. Staring, L.W. Molenkamp, B.W. Alphenhaar, H. van Houten, O.J.A. Buyk, M.A.A. Mabesoone, C.W.J. Beenakker, and C.T. Foxon, Europhys. Lett. 22, 57 (1993).

${ }^{9}$ A.S. Dzurak, C.G. Smith, C.H.W. Barnes, M. Pepper, L. Martin-Moreno, C.T. Liang, D.A. Ritchie, and G.A.C. Jones, Phys. Rev. B 55, R10197 (1997).

${ }^{10}$ S. Möller, H. Buhmann, S.F. Godijn, and L.W. Molenkamp, Phys. Rev. Lett. 81, 5197 (1998).

${ }^{11}$ M. Turek and K.A. Matveev, cond-mat/0112445, to be published in Phys. Rev. B; K.A. Matveev, in Statistical and Dynamical Aspects of Mesoscopic Systems, ed. by D. Reguera, G. Platero, L.L. Bonilla, and J.M. Rubi (Springer, Berlin, 2000), p. 3.

${ }^{12}$ K. Flensberg, Phys. Rev. B 48, 11156 (1993); Physica (Amsterdam) 203B, 432 (1994).

${ }^{13}$ K.A. Matveev, Phys. Rev. B 51, 1743 (1995).

${ }^{14}$ A. Furusaki and K.A. Matveev, Phys. Rev. B 52, 16676 (1995).

${ }^{15}$ I.L. Aleiner and L.I. Glazman, Phys. Rev. B 57, 9608 (1998).

${ }^{16}$ H. Yi and C. L. Kane, Phys. Rev. B 53, 12956 (1996).

17 A.V. Andreev and K.A. Matveev, Phys. Rev. Lett. 86, 280 (2001). 